\title{
On-Chip Integrated Optical Sensors for Fluorescence Detection of Cancer Tissue: Application to Capsule Endoscopy
}

\author{
R. A. Dias, J. H. Correia, G. Minas \\ Dept. of Industrial Electronics, Laboratory of Biomedical Engineering \\ University of Minho \\ Campus de Gualtar, 4710-057 Braga, Portugal \\ rosana@dei.uminho.pt, higino.correia@dei.uminho.pt,gminas@dei.uminho.pt
}

\begin{abstract}
Human tissues show autofluorescence emission spectra when excited by ultraviolet or short-wavelength visible light. The intensity and shape of these spectra depends of the tissues pathologic state. Therefore, its measurement can give information about the degree of malignant transformations that could lead to cancer. This paper describes the design of an integrated CMOS compatible fluorescence measurement system that exploits those emission spectra differences. It comprises optical sensors, optimized for measuring the fluorescence intensity at specific spectral bands, and readout electronics for data processing. This system can be integrated in an ingestible electronic capsule used for monitoring physiological parameters of the gastrointestinal tract.
\end{abstract}

\section{INTRODUCTION}

Early stage cancer detection is a major goal in medicine, being the most important factor on which the patient's survival rate depends. Some of the most promising methods for detecting cancer at an early stage are based on tissue's fluorescence. The abnormal tissue absorbs and emits light at specific wavelengths differently from the normal tissue, due to differences on endogenous chromophores and fluorophores concentrations $[1,2]$. The capsule endoscopy (EC) may play an important role in early stage cancer detection, once it can be equipped with miniaturized diagnosis and treatment instruments, such as the fluorescence biosensor discussed in this paper.

The capsule endoscopy is a relatively recent method for gastrointestinal (GI) tract evaluation. It consists, basically, of a swallowable capsule, with a CCD (Charge Couple Devices) or CMOS (Complementary Metal Oxide Semiconductor) imager and two pairs of white LEDs (Light Emitting Diodes) [3, 4]. It takes approximately 2 pictures per second as it travels due to the peristaltic movements $[3,4]$. The endoscopic capsule (EC) sends the obtained images to the outside of the organism through a radio-frequency signal, which is externally detected [4]. Therefore, the diagnosis depends only on those images and on the video file built from them, although capsules with lab-on-a-chip systems

Support for this research was provided by R\&D Centre Algoritmi of University of Minho. that measure $\mathrm{pH}$, temperature and pressure are, nowadays, starting to be a reality. Reading and interpreting the images takes around 1-2 hours, which is too long considering that the artifacts found may concern to only a small fraction of the total analyzed data $[4,5]$. To reduce CE limitations and enhance the diagnostic yield, besides the obvious improvements of the EC components, especially the ones related to the image quality, there are mainly three research fields: (1) the image processing techniques that may be applied to the images for helping detect certain artifacts based, for example, on pigment and shape information; (2) the developing of locomotion and stop mechanisms to incorporate on the EC for being stopped at a specific site an return there if necessary; and (3) providing the capsule with sensors and tools to detect and actuate on abnormalities, whose usefulness depends on the EC stop mechanisms and maneuverability. This report fits in the latter research field.

This paper reports the project of optical sensors capable of evaluating, by reading the fluorescence emission spectra of a tissue, the tissue's blood irrigation and detecting possible neoplastic lesions. These miniaturized sensors were designed to be able of being incorporated on the EC.

\section{FluORESCENCE DETECTION OF CANCER TISSUE}

Epithelial tissue presents autofluorescence (AF) when excited by ultraviolet or short-wavelength visible light. In this light condition, certain biomolecules mainly the ones in the submucosa of the epithelium, known as endogenous fluorophores, emanate light at longer-wavelengths visible light [1]. The emission wavelength range depends on the different groups of fluorophores that are activated. As an example, collagen and elastin emit on the green spectral range; porphyrin emits on the red spectral range; etc [6].

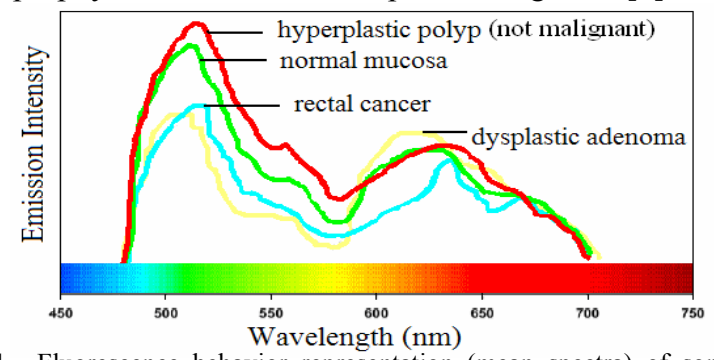

Figure 1. Fluorescence behavior representation (mean spectra) of some colorectal tissues. 
In the ultraviolet and visible part of the spectrum, the optical properties of the tissue are dominated by the endogenous chromophores (light-absorbing biomolecules), of which the most common is haemoglobin $(\mathrm{Hb})$. The chromophores types, distribution and concentration are different for normal and abnormal tissue and have different fluorescent spectra as well [2]. Since neoplastic tissue (tissue with formation of tumors) has higher blood irrigation (meaning more $\mathrm{Hb}$ concentration) and it is usually thicker than normal tissue, less light is transmitted through the mucosa to the submucosa. Thus, less light excites the endogenous fluorophores present in the submucosa. As a consequence, less fluorescent light (emitted by those fluorophores) is transmitted through the neoplastic tissue for detection, once $\mathrm{Hb}$ absorbs most of the light [6]. Therefore, the AF intensity of epithelial tissue with adenocarcinoma, inflammation or other high blood-irrigation is lower than normal tissue and it can be used to distinguish the presence and absence of malignant lesions. Usually, the spectra from normal and abnormal tissue are similar, e. g., both have two characteristic peaks, one in the green range and another in the red range (see Fig. 1). However, in normal tissue, the intensity of the green peak is much higher than the intensity of the red peak. In contrast, in abnormal tissue, the intensity of the green peak decreases (when compared to the one of the normal tissue) getting closer or even lower than the intensity of the red peak (see Fig. 1). It is also important to notice that, in the case of normal mucosa, if the excitation light is very intense and the tissue sample is thick, the green fluorescence peak can even camouflage the red peak.

Mayinger et al. performed light-induced autofluorescence spectroscopy in-vivo on the esophagus, the stomach and the colorectum [6-9]. They established an algorithm to distinguish (pre)-cancerous lesions from normal mucosa. This algorithm is based on the shape variations that are observed between the spectra from both types of tissue. Based on their results, the most representative wavelengths for the fluorescence peaks are around $515 \mathrm{~nm}$ and $620 \mathrm{~nm}$. Therefore, reading the intensity of those peaks and making calculations, based on spectral analysis, it can be obtained information about the degree of malignant tissue transformations. However, in terms of what can be visualized through the endoscope, Uedo et al. in [10] showed that a superficial neoplasm has a similar appearance to inflamed tissue on AF imaging, and they could be misunderstood. A green reflectance measurement might be the solution to overcome this problem, since $\mathrm{Hb}$ preferably absorbs green light in comparison to tumor tissue [10]. The optical detection microsystem reported here exploits both those features, once it includes selective optical interference filters optimized for each spectral range (Fig. 2).

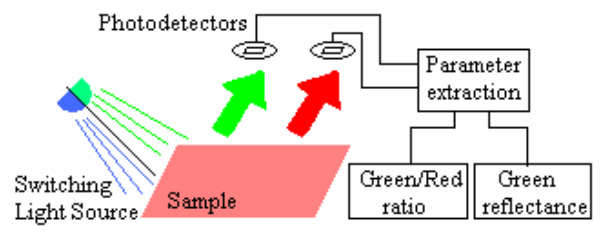

Figure 2. Basic functioning principle of the optical biossensor.

\section{OptiCAL Microsystem DESIGN AND OPERATION}

\section{A. Optical Detectors}

The optical sensors should be incorporated in an EC for measuring the tissue autofluorescence emission spectra. Moreover, they should have integrated in the same die their readout circuits for converting the analog photodetectors signal into a digital signal, with a suitable format for RF transmission. Merging the optical sensors with other functions on a single-chip means that the system would have to fit in a standard microelectronic process, preferably CMOS. The most common structure for photodetectors in standard CMOS is the junction photodiode, which is simply a $p n$-junction located at some distance from the surface. If it is illuminated from the front side, the deeper the junction, the higher the efficiency in collecting longer wavelengths will be.

There are two basic opportunities for changing the spectral response of photodiodes in standard CMOS process: (1) selecting the suitable junction depth and (2) selecting a suitable combination of the dielectric layers that may be present on top of the photodetector, acting as interference filters. However, in a standard CMOS process the junction depth is fully defined and cannot be altered. Fortunately, the process provides three photosensitive structures as vertical junction photodiodes: $n$-well/p-epilayer, $n+/ p$-epilayer and $p+/ n$-well [11]. At a particular wavelength, their quantum efficiency varies according to their junction depth. The quantum efficiency of a photodiode reveals how well it collects the incident light [12]. In the visible spectrum, the blue light (of about $450 \mathrm{~nm}$ ) is more efficiently collected by a shallower junction ( $p+/ n$-well and $n+/ p$-epilayer $)$ and the red light (of about $650 \mathrm{~nm}$ ) by a deeper junction (n-well/p-epilayer). Moreover, despite the shallower junction of the $n+/ p$-epilayer photodiode, it has the higher quantum efficiency in the wavelengths of about $500 \mathrm{~nm}$, due to the different doping concentration between the $n$ and the $p$ side, which extends the $p$ side depletion area more deeply. Therefore, the photodiodes used in the reported work are the $n+/ p$-epilayer (Fig. 3a) for collecting the green light and the $n$-well/p-epilayer (Fig. $3 b$ ) for collecting the red light. They were fabricated in a $0.7 \mu \mathrm{m}$, double-metal, $n$-well CMOS process. Each photodiode has a $100 \mu \mathrm{m} \times 100 \mu \mathrm{m}$ active area. Their measured quantum efficiency is shown in Fig. 4.

The second opportunity for changing the photodiodes spectral response is to play with the stack of dielectric layers possibly left on top of the photodiode $p n$-junction. In the standard double-metal CMOS process used, there are three dielectric layers above the $p n$-junction. Simulations show that the dielectric layers can increase the quantum efficiency at certain wavelengths but it also introduces wavelength dependence [13]. Therefore, the photodiode structure without two of those three dielectric layers provides the best possible quantum efficiency in the visible spectral range. The first dielectric layer should be left over due to fabrication constraints. Fig. 4 shows the measured effect of the dielectric layers on top of the $n+/ p$-epilayer photodiode. 


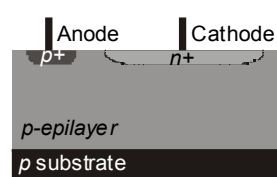

(a)

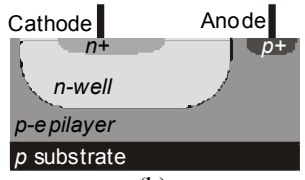

(b)
Figure 3. The two junction photodiodes used: (a) $n+/ p$-epilayer and (b) n-well/p-epilayer.

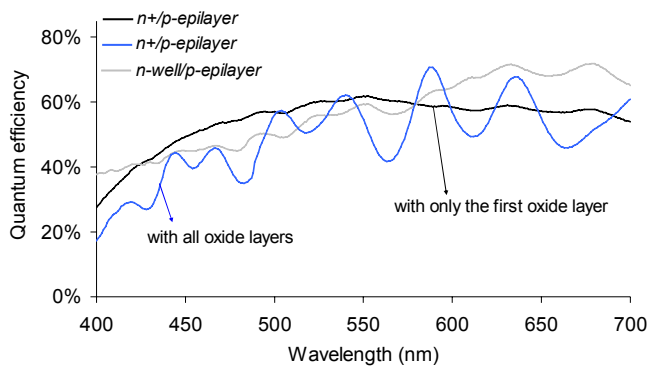

Figure 4. Measured quantum efficiency of the two fabricated fotodiodes and effect of the dielectric layers on top of the $n+/ p$-epilayer photodiode.

\section{B. Optical Filters}

The photodetectors optical response can be improved if two thin-film optical filters, with a passband around the green $(510$ to $520 \mathrm{~nm})$ and the red (610 to $620 \mathrm{~nm})$ light, are placed on top of each photodiode active area. The optical interference filter is based on a Fabry-Perot thin-film optical resonator [14] designed for being fabricated in a compatible CMOS post-process. A thin-film software package $\left(\mathrm{TFCalc}^{\mathrm{TM}}\right)$ was used for the structural optimization of the optical filters together with the transmission through the dielectric layer on top of the $p n$-junction provided by the standard CMOS process. Simulation results show that a layer stack of $40 \mathrm{~nm} \mathrm{TiO}_{2}$ and $95 \mathrm{~nm} \mathrm{SiO}_{2}$ for the dielectric mirrors with a $\mathrm{SiO}_{2}$ resonance cavity is the best option in terms of optical characteristics and feasibility (Fig. 5). The thickness of the resonance cavity is $180 \mathrm{~nm}$ and $245 \mathrm{~nm}$ for collecting the green and the red light, respectively. The optical filters are post-processed, by evaporation, using only one additional mask for the resonance cavity thicknesses. Besides they provide an optimized optical response at the two autofluorescence tissue emission peaks, they also avoid interference peaks in the neighborhood wavelengths caused by optical events (such as Raman effect) that could lead to wrong fluorescence intensity measurements. Moreover, with this scheme it might be possible to distinguish a neoplasm from an inflammation.

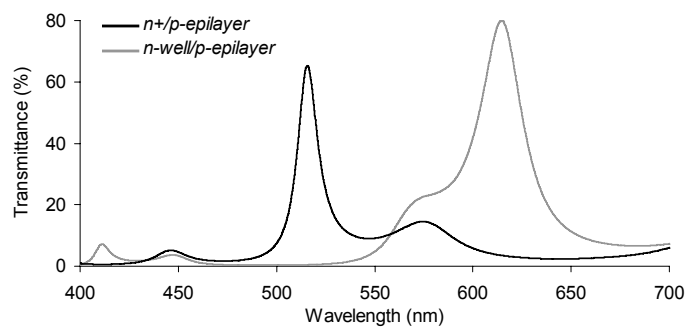

Figure 5. Simulated spectral transmittance of the optical filters (optical effect of fabricated photodiode included).

\section{Readout Electronics}

The basic principle used for malignant tissue detection at an early stage is based on accurate measurement of the photocurrents and the photocurrents ratio provided by the two photodetectors: the $n+/ p$-epilayer that collets the green light and the n-well/p-epilayer that collets the red light. A high ratio will mean the green peak is much higher than the red one (healthy tissue feature). The very high linearity of silicon photodiodes makes this current ratio dependent on the spectral information and not on the light intensity. A reference signal (Fig. 6) of this current ratio for the same light wavelength is included in the readout electronics for normalization. This signal was obtained using the measured quantum efficiency of both photodiodes. Moreover, two additional photodiodes completely covered with metal for measuring the photodiode dark current are also introduced in the circuit. The dark current is usually measured and subtracted from the flux. Since it is temperature dependent, one measurement at the beginning of the experiment is usually not sufficient. Thus, in the reported circuit, a dark current compensation channel is implemented.

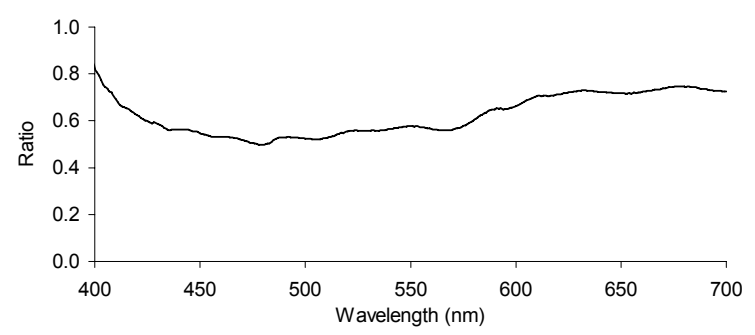

Figure 6. Measured current ratio of the $n+/ p$-epilayer and the $n$-well/p-epilayer photodiodes.

The on-chip analog to digital conversion is performed using a sigma-delta converter for each photodiode. It produces a bit stream signal and allows its use in small dataacquisition and control systems. The oversampling principle allows a flexible trade-off between bandwidth and accuracy (noise). Since the bandwidth of the CMOS circuits is much higher than the required bandwidth for the light measurements, a simple first-order sigma-delta converter with a high oversampling ratio can be used. Moreover, it can be performed with very little silicon area. The digital values from the sigma-delta are reconstructed using a decimation filter, which can be programmed in a microcontroller. The sigma-delta output signal is proportional to the photodiode current and hence proportional to the fluorescent light emitted by the tissue.

\section{FLUORESCENCE MEASUREMENTS}

To calibrate and improve the optical detection microsystem, some experimental measurements need to be performed. The experimental results were obtained using pig's large intestine tissue samples. The samples were classified according tissue blood irrigation, evaluated through naked eye, by coloration, as highly irrigated (HI) or with low irrigation (LI). The AF emission spectra were obtained using a SPEX $16800.22 \mathrm{~m}$ Double Spectrometer, a SPEX FLUOROLOG, a $300 \mathrm{~W}$ Xenon lamp as light source 
and readout electronics for signal conditioning (Fig. 7). Several excitation wavelengths between $300 \mathrm{~nm}$ and $490 \mathrm{~nm}$ were tested for selecting the wavelength that better excites the tissues. As the spectral shapes obtained were comparable, the measurements on pig's large intestine were performed at $400 \mathrm{~nm}$ excitation light. The measured data were processed for removing equipment artifacts (a Gaussian spectral analysis was also included in this processing). Fig. 8 shows the autofluorescence emission spectra. It can be seen the decrease in green fluorescence and the increase in red, with change from LI to HI tissue. Therefore, the ratio between mean intensity in the $510-530 \mathrm{~nm}$ range and the mean intensity in the $600-650 \mathrm{~nm}$ range may provide a good parameter for a first approximation in the characterization of the tissue.

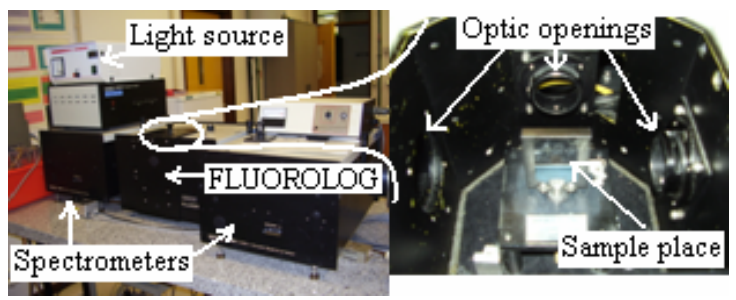

Figure 7. Experimental setup.

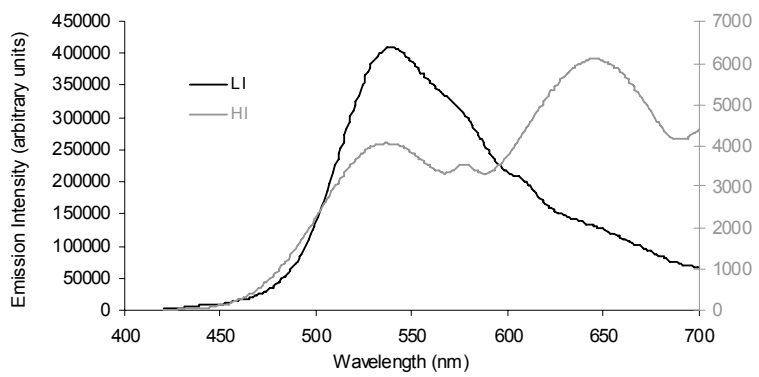

Figure 8. Measured autofluorescence emission intensity on LI and HI samples at an excitation wavelength of $400 \mathrm{~nm}$.

\section{CONCLUSIONS}

The on-chip integrated CMOS optical sensors presented here have been designed and optimized for being integrated in an endoscopic capsule for fluorescence detection of malignant tissues. The autofluorescence emission spectra intensity and shape of a tissue is dependent of the tissue pathologic state at specific spectral bands. The optical sensors measure fluorescence intensity and, their output, after being processed by readout electronics, is used for obtaining the green/red fluorescence ratio intensity. This parameter may give information about the degree of malignant lesions. For this purpose, the optical CMOS photodetectors include a set of thin-film layers, post-processed on their top, to form a selective passband optical filter, optimized for the green and the red spectral bands only. This scheme helps to distinguish a superficial neoplasm from an inflammation. The measurements results on ex-vivo pig's large intestine have shown promising performance and a starting point for human tissue's analysis with several cancer stage developments. A green reflectance can be used to distinguish inflamed from cancerous tissue, therefore it should also be measured. Meanwhile, measurements using blue (to induce fluorescence) and green (to obtain reflectance) LEDs as excitation light source are on-going in order to incorporate it on the EC.

Presently, only the photodetectors (not yet with the thinfilms filters) are fabricated. In the future, a prototype must be developed and tests must be performed with in vivo cancerous lesions and inflamed tissue samples in order to validate this system's efficiency and the significance of the parameters obtained.

\section{ACKNOWLEDGEMENTS}

The authors wish to acknowledge Paulo Coutinho, from Dept. of Physics, University of Minho, for his help with the signals spectral analyses.

\section{REFERENCES}

[1] R.S. DaCosta, B.C. Wilson, and N.E. Marcon, "Spectroscopy and fluorescence in esophageal diseases," Best Practice \& Research Clinical Gastroenterology, vol. 20, pp. 41-57, February 2006.

[2] M. Stringer and K. Moghissi, "Photodiagnosis and fluorescence imaging in clinical practice," Photodiagnosis and Photodynamic Therapy, vol. 1, pp. 9-12, May 2004.

[3] D. Fleisher, "Capsule imaging”, Clinical Gastrology and Hepatology, vol. 3, pp. s30-s32, July 2005.

[4] M. Delvaux and G. Gay, "Capsule endoscopy in 2005: facts and perspectives," Best Practice \& Research Clinical Gastroenterology, vol. 20, February 2006, pp. 23-39.

[5] D. R. Deal, L. Lambiase and J. Li, "M2A capsule endoscopy," Jacksonville Medicine, 2002. Available on-line at http://www. dcmsonline.org/jax-medicine/2002journals/junejuly2002/capsule.htm.

[6] B. Mayinger, et al., "Light-induced autofluorescence spectroscopy for tissue diagnosis of GI lesions," Gastrointestinal Endoscopy, vol. 52, pp. 395-400, September 2000.

[7] B. Mayinger, et al., "Evaluation of in vivo endoscopic autofluorescence spectroscopy in gastric cancer," Gastrointestinal Endoscopy, vol. 59, pp. 191-198, February 2004.

[8] B. Mayinger, et al., "Endoscopic light-induced autofluorescence spectroscopy for the diagnosis of colorectal cancer and adenoma," Journal of Photochemistry and Photobiology B: Biology, vol. 70, pp. 13-20, April 2003.

[9] B. Mayinger, et al., "Light-induced autofluorescence spectroscopy for the endoscopic detection of esophageal cancer," Gastrointestinal Endoscopy, vol. 54, pp. 195-201, August 2001.

[10] N. Uedo, et al., "A novel videoendoscopy system by using autofluorescence and reflectance imaging for diagnosis of esophagogastric cancers," Gastrointestinal Endoscopy, vol. 62, pp. 521-528, October 2005.

[11] P. Aubert, H. Oguey, and R. Vuillemier, "Monolithic optical position encoder with on-chip photodiodes," IEEE J. Solid-State Circuits, vol. 23, pp. 465-472, 1988.

[12] S. M. SZE, Physics of semiconductors devices, $2^{\text {nd }}$ ed., John Wiley \&Sons, New York, 1981, pp. 74-79.

[13] G. Minas, J.C. Ribeiro, R.F. Wolffenbuttel and J.H. Correia, "On-Chip integrated CMOS optical detection microsystem for spectrophotometric analyses in biological microfluidic systems," Proceedings of ISIE 2005, pp. 1133-1138, June 2005.

[14] G. Minas, R. F. Wolffenbuttel and J. H. Correia, “An array of highly selective Fabry-Perot optical-channels for biological fluids analysis by optical absorption using white light source for illumination," J. Opt A: Pure and Appl Opt, vol. 8, pp. 272-278, 2006. 